\title{
Undetected changes in visible stimuli influence subsequent decisions
}

\author{
Cédric Laloyaux ${ }^{1 *}$, Christel Devue ${ }^{2}$, Stéphane Doyen $^{1}$, Elodie David $^{1}$, and \\ Axel Cleeremans ${ }^{1}$
}

1: Cognitive Science research Unit

Université Libre de Bruxelles (U.L.B.) CP 191

Av. F.-D. Roosevelt, 50

1050 Bruxelles

BELGIUM

Tel: +3226504231

Fax: +3226502209

Email: claloyau@ulb.ac.be

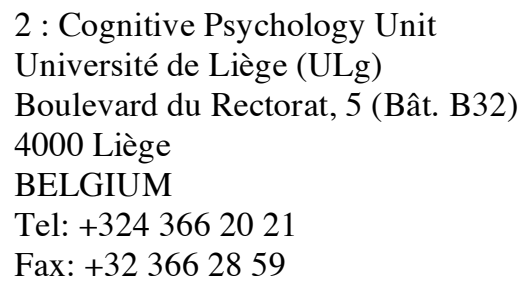

Keywords: change blindness, change detection, implicit change detection, consciousness, awareness, visual memory

$$
\text { * Corresponding author }
$$




\begin{abstract}
Change blindness - our inability to detect changes in a stimulus-occurs even when the change takes place gradually, without any disruption (Simons et al., 2000). Such gradual changes are more difficult to detect than changes that involve a disruption. Using this method, David et al. (in press) recently showed substantial blindness to changes that involve facial expressions of emotion. In this experiment, we show that people who failed to detect any change in the displays were (1) nevertheless influenced by the changing information in subsequent recognition decisions about which facial expression they had seen, and (2) that their confidence in their decisions was lower after exposure to changing vs. static displays. The findings therefore support the notion that undetected changes that occur in highly salient stimuli may be causally efficacious and influence subsequent behaviour. Implications concerning the nature of the representations associated with undetected changes are discussed.
\end{abstract}




\section{Introduction}

Change blindness, our inability to detect large changes in visual displays, is a striking phenomenon that has now been demonstrated through various paradigms (e.g. Rensink, 2002; Rensink, O'Regan, \& Clark, 2000; Rensink, ORegan, \& Clark, 1997; Simons, 2000; Simons \& Levin, 1998; Simons \& Levin, 2003).

While early empirical work in this domain was characterized by the use of highly artificial stimuli consisting of dot matrices (Phillips, 1974) or letter arrays (Pashler, 1988), more recent demonstrations involve changes that occur in complex, realistic scenes (Rensink et al., 2000; Rensink et al., 1997; Simons, Franconeri, \& Reimer, 2000; Velichkovsky, Dornhoefer, Kopf, Helmert, \& Joos, 2002). Change blindness challenges introspective judgments that our perception of the world is complete and accurate-a belief so strongly held that it has been dubbed "change blindness blindness" (Levin, Momen, Drivdahl, \& Simons, 2000). For instance, in real life situations, observers fail to notice changes as dramatic as switching an individual with whom they are interacting to a different person (Levin \& Simons, 1997). Moreover, observers continue to exhibit change blindness even when directly instructed to detect changes (Rensink et al., 2000; Rensink et al., 1997; Simons et al., 2000). Numerous relevant studies have used the so-called "flicker" paradigm, in which two images, identical to each other but for a single change, are displayed alternatively for $240 \mathrm{~ms}$ and separated from each other by a $80 \mathrm{~ms}$ blank screen - the disruption. This flickering sequence is typically repeated until the observer detects the change. It can take many such cycles (up to hundreds in some conditions) for 
observers to become aware of the change (Rensink et al., 1997). Rensink et al. (1997) have documented that detection rate is influenced by the location at which the change occurs. For instance, performance improves when the change involves an area of major interest compared to an area of marginal interest.

In such flicker studies (Rensink et al., 2000; Rensink et al., 1997; Simons et al., 2000), change blindness is induced by a brief visual disruption inserted between the two target images, and hence the demonstration is perhaps less compelling than it might first appear because the images only appear for a brief duration. "Gradual change" studies address this limitation by introducing changes only very progressively (e.g., over a period of 12 seconds), without any disruption. With this method, observers have the opportunity to look at the image carefully ${ }^{1}$ and in an uninterrupted manner. Studies using this paradigm have explored people's ability to detect changes in object color or changes involving the deletion or addition of an object. Simons et al. (2000) showed that gradual change produces powerful change blindness despite the fact that the change is continuously happening in front of the observer. However, change blindness rate was lower under gradual change conditions than under disruption conditions for changes involving deletion or addition. However, as Simons et al. (2000) pointed out, this result might be due to artifacts. Indeed, gradual changes, when they involve deletion or addition, necessarily produce intermediate frames that contain easily noticed artifacts, such as transparent objects (Simons et al., 2000). In a second experiment that avoided such artifacts by using color changes, Simons et al. (2000) showed that change blindness

\footnotetext{
${ }^{1}$ Note that Hollingworth used long pre-change display presentation (up to $20 \mathrm{sec}$ ) to address this limitation (Hollingworth, 2003, 2004)
} 
rate was higher for gradual changes than for disruption changes (see also Auvray \& O'Regan, 2003 for similar conclusions).

In this light, David, Laloyaux, Devue and Cleeremans (in press) ${ }^{2}$ investigated gradual changes with facial expressions of emotions because expression changes involve essentially modifications to the shape and spatial relationships of features that are internal to an object rather than the appearance or disappearance of new objects. They showed that such changes still produce quite substantial rates of change blindness, as only about $15 \%$ of participants detected slow, gradual changes in the facial expression of actors, even when such changes occur in front of their eyes as they intentionally scrutinize the stimuli under direct instructions to detect any changes.

These and other findings leave open an important question, however: What is represented, if anything, when a change remains undetected? Authors have suggested several explanations (see, Simons, 2000, for a review). A first possibility is that there is a failure to encode or represent the pre-change and/or post-change information (O'Regan \& Noe, 2001). This absence of visual representation would explain change blindness because comparison with the post-change information is then be impossible. Another possibility is that the representation of the pre-change information is erased or overwritten by the post-change information (Beck \& Levin, 2003; Becker, Pashler, \& Anstis, 2000; Landman, Spekreijse, \& Lamme, 2003; Tatler, 2001). Other authors have suggested that it is also possible that while information about both pre- and post-change

\footnotetext{
${ }^{2}$ An example stimulus is available at the following URL: http://srsc.ulb.ac.be/DemoLaloyauxetal.mov
} 
states is stored in some manner, the comparison between the two representations fails, which would likewise produce change blindness (Angelone, Levin, \& Simons, 2003; Hollingworth \& Henderson, 2002; Mitroff, Simons, \& Levin, 2004; Scott-Brown, Baker, \& Orbach, 2000; Silverman \& Mack, 2006; Simons, Chabris, Schnur, \& Levin, 2002). In this latter case, the problem is thus not about representations, but rather about an operation that has to be performed on them.

Simons (2000) usefully summarized 5 alternatives as follows: (1) “Overwriting", which means that pre-change representation would be erased by post-change representation (Beck \& Levin, 2003; Becker et al., 2000; Landman et al., 2003; Tatler, 2001), (2) "First impression", which suggests that it would only be the pre-change representation that is represented and not what is presented after the change. This account makes it difficult, however, to explain why the visual system would stop forming representations when a change is displayed, as representations are updated with time (Hollingworth \& Henderson, 2004), (3) "Nothing is stored" is a position defended by O'Regan and Noë (2001), and which posits that no visual representation should be postulated to perceive the world. (4) "Everything is stored but nothing is compared" assumes that some representation is formed both for the pre-change and the post-change display but that no comparison is made between both representations when change blindness occurs. As noted earlier, much recent evidence suggests that this possibility might explain some change blindness cases (Angelone et al., 2003; Hollingworth \& Henderson, 2002; Mitroff et al., 2004; Scott-Brown et al., 2000; Silverman \& Mack, 2006; Simons et al., 2002). Finally, (5) "Feature combination" suggests that an integrated 
representation would be formed based on both pre- and post-change displays, which would then make change detection impossible.

Leaving aside for the time being the question of which of these accounts is most likely to be correct, the question of whether undetected changes are represented somehow was directly addressed in a convincing study by Mitroff et al. (2004), who showed that observers remain capable of recognizing, in a forced-choice task, both the pre and postchange information, even when reporting being unaware of having detected a change. In that study, observers were exposed to changes in a classic change detection paradigm (Phillips, 1974) in which 4 to 8 objects were displayed on a computer screen. On most trials, one of the objects changed into another object. Right after the change detection trial, a series of subsequent two-alternative forced-choice (2AFC) trials was administered in random order. One $2 \mathrm{AFC}$ trial presented the pre-change object along with a random novel object that had not been presented in the trial, and participants were asked to choose which had been presented. Another 2AFC trial presented the post-change object along with a random novel object. A third $2 \mathrm{ACF}$ trial presented a random presented nonchanging object along with a novel object. Observers were asked to report if they had detected the change after (Experiments 1-3) or before (Experiment 4) the series of 2AFC trials. Through this design, Mitroff et al. showed that even when subjects were unaware of the change, they were nevertheless able to remember, better than expected by chance, both the pre- and post-change information. 
However, the question of determining what representation observers form of the material when exposed to changing stimuli that contain more than two images has never been explored directly. When only two images (i.e., the pre- and the post-change displays) are presented, as in the flicker paradigm, subjects may remember the prechange display, the post-change display, or both, as shown by Mitroff et al. But what is remembered when many intermediate images are presented, as in the gradual change paradigm? Do participants remember only the first or the last display? Or do they construct some average representation of the entire sequence? These are essentially the questions we explored in this study.

Instead of testing every aspect of the representation observers have formed of a changing stimulus, which is impractical, we asked what stimulus subjects spontaneously select when asked to identify what they had seen among a limited number (i.e., five) of perceivably different images. Crucially, in our paradigm, participants were not informed that a change may occur, and we focused data analysis on those participants who had remained unaware of the fact that some stimuli were changing. This is important for previous studies (Angelone et al., 2003; Hollingworth \& Henderson, 2002; Mitroff et al., 2004; Scott-Brown et al., 2000; Silverman \& Mack, 2006; Simons et al., 2002) have demonstrated that some information both about pre and post-change displays is stored when observers are forewarned that a change may occur

This issue is also related to recent debates about implicit change detection (Fernandez-Duque, Grossi, Thornton, \& Neville, 2003; Fernandez-Duque \& Thornton, 
2000, 2003; Laloyaux, Destrebecqz, \& Cleeremans, 2006; Mitroff, Simons, \& Franconeri, 2002; Thornton \& Fernandez-Duque, 2000, 2002). For instance, FernandezDuque and Thornton (2000) presented their subjects with changes of orientation of horizontal and vertical rectangles and showed that, even when subjects reported being unaware of a change, they were nevertheless able to localize the change above chance level. In a different paradigm, Thornton and Fernandez-Duque (2000) again presented their subjects with horizontal and vertical rectangles but showed that exposure to a change in the orientation of an item produced a congruency effect in a subsequent judgment task about the orientation of a rectangle. Although these studies were criticized by Mitroff et al. (2002), it seems that these effects persist even after the correction of potential biases (Fernandez-Duque \& Thornton, 2003; Laloyaux et al., 2006). It therefore seems that a change that observers report as being unaware of can influence subsequent behavior.

This question, however, remains open for complex stimuli that involve more than 2 subsequent brief displays of rectangles. A recent study (Hollingworth \& Henderson, 2004) is relevant in this context. The authors used what could be called a "gradual flicker paradigm", wherein a scene was progressively rotated in steps of 1 degree of visual angle on each display, with each display separated from the next one by a brief blank screen. The results indicated that half of the participants were unaware of the change up to a rotation of $48^{\circ}$. However, when a shift (using a blank to avoid low level signal) back to the original scene was produced after a cumulated rotation of 20 or $30^{\circ}$ that had remained undetected, most subjects could then clearly see the change, suggesting the existence that 
visual memory had been updated unconsciously. This conclusion follows from the fact that had visual memory not been udpated at all, subjects would not have been able to detect the change when coming back to the original display.

In the present study, we thus explored the same issue, but using a fully continuous stimulus, and a different reporting methodology that allows for somewhat stronger conclusions. Specifically, in this experiment, we used the subset of stimuli involving gradual changes of facial expressions used by David at al. (in press) to investigate what representation observers form when exposed to a change while remaining unaware of this change. If nothing at all is represented, then one should expect to observe a completely flat distribution of recognition choices, that is, observers should choose all of the intermediate images equally often. It should be noted that the same prediction can be made if "everything is represented". However, if the distribution of choices differs from pure chance, (e.g., if it exhibits a primacy or recency effect, or if some other bias towards particular intermediates is apparent), this would suggest unconscious sensitivity to change, or at least an update of the representation during the change. It is important to realize that finding a distribution of choices that differs from a flat distribution rules out both the "nothing is represented" hypothesis and the "everything is equally represented" hypothesis.

Note that the task is prima facie identical for static and for changing stimuli, that is, it is just as easy for people to express their choice after having been exposed to a changing or to a static stimulus, given that we only considered participants who had 
remained unaware of the changes. In other words, all participants whose results we analyze below think that they had been exposed to static, photographic stimuli.

To summarize, in this experiment, we used the subset of stimuli involving gradual changes of facial expressions used by David at al. (in press) to investigate what representation observers form when exposed to a change while remaining unaware of this change.

\section{Method}

Subjects. 49 undergraduate students from the Université Libre de Bruxelles participated either for partial class credit or for 4 Euros.

Materials. The material was composed of the 8 stimuli involving facial expression changes used in David et al. (in press). David et al. used 8 different scenes composed of 3 actors (three different actors for each scene) showing various facial expressions and placed in different locations. The scenes were either static, or they contained a facial expression change based on two different snapshots (see Fig. 1 for an example). The modified facial expression was "pasted" carefully on the original scene. Using morphing software (Morph Man 2000), 144 intermediate frames for each AB pair were then created and assembled in a 12s QuickTime movie. Facial expression changes represented an average of $4.2 \%$ of the total surface of the image. For each pair of images, we created two movies, one involving a Neutral (N) to Emotional (E) change, and the second involving an $\mathrm{E}$ to $\mathrm{N}$ change. David et al. showed that such changes were detected only by $15 \%$ of 
participants when explicitly required to detect them under conditions where they were also presented with color changes.

Insert Fig. 1 around here

Stimuli were displayed on a 17" CRT screen at a resolution of 1024 by 768 pixels. Each scene had a size of 756 by 567 pixels (subtending about $19^{\circ}$ by $25^{\circ}$ of visual angle) and was presented on a gray background. Subjects were tested either individually either in groups of up to 8 participants in the same room (in which case each participant was isolated from the others in a single box and wearing headphones playing white noise).

In addition, new material was created for the recognition task: for each target face, a square was isolated from the whole scene image and resized to an image of $150 * 150$ pixels, substending $4 * 4$ degrees. Thus, as the size of the target face was different in each of the scenes (see David et al., for a complete description of the material), a square that framed the face was created and this square was isolated from the rest of the image. The square was then resized to $150 * 150$ pixels if necessary so that all recognition stimuli were identical in size. Moreover, 5 morphs (Figure 2) ranging between an $\mathrm{E}$ and $\mathrm{N}$ expression $(0 \% \mathrm{~N}-100 \%$ E, $25 \% \mathrm{~N}-75 \%$ E, $50 \% \mathrm{~N}-50 \% \mathrm{E}, 75 \% \mathrm{~N}-25 \% \mathrm{E}, 100 \% \mathrm{~N}$ $-0 \%$ E) were created (again using Morphman 2000) and displayed on the screen aligned in a random order during the recognition task. Thus, for each target face, we first took the two most extreme faces: $\mathrm{E}$ and $\mathrm{N}$. These two were respectively the " $0 \% \mathrm{~N}-100 \% \mathrm{E}$ " face and the "100\% E - 0\% N" face. Next, we created 3 intermediates between these two 
extreme pictures: one with $25 \%$ of emotional facial expression and $75 \%$ of neutral facial expression " $25 \% \mathrm{~N}-75 \% \mathrm{E}$ ", a second that consisted of an equal mixture of the two expressions, "50\% $\mathrm{N}-50 \% \mathrm{E}$ ", and finally a third with $75 \%$ of emotional facial expression and $25 \%$ of neutral facial expression " $75 \% \mathrm{~N}-25 \%$ E". Thus we ended up with 5 stimuli, each depicting facial expressions ranging from $\mathrm{E}$ to $\mathrm{N}$ and that were shown to observers in a random order on the screen.

Procedure. Participants were instructed to memorize the faces "because they would be subsequently asked to recognize them by selecting the exact faces observer had been exposed to." Observers were unwittingly exposed to 4 changing stimuli (movies) together with 4 non-changing (static) stimuli for $12 \mathrm{~s}$ (see Figure 2). Presentation order of the 8 stimuli (movie or static) was randomized. The four changing stimuli contained gradual changes that occurred over a period of 12 seconds. Half of them contained positive emotional expressions and the other half contained negative emotional expressions; half began with the Neutral expression and half began with the Emotional expression.

Insert Fig. 2 around here

After each presentation, participants were asked to select the image they had been exposed to among the 5 morphs described above, and to rate their confidence in their decision on a 4 -points scale $(4=$ very high, $3=$ high, $2=$ low, $1=$ very low $)$. At the end of the experiment, observers were asked if they had noticed "anything unusual" and 
"anything that had changed" in the stimuli so as to ascertain whether they were aware of the occurrence of changes in some of the stimuli. We asked them 4 questions that became more and more explicit about a change. If subjects reported any sense of anything "strange" or "unusual", we considered them as being aware of the change, thus using a very conservative criterion.

\section{Results}

Fourteen out of forty-nine participants reported awareness of at least one change. They were thus considered "aware" and discarded from the analysis, which thus concerned "unaware" participants only. Thus, 35 out of 49 (71.4\%) participants failed to detect any change and were included in the analysis.

Insert Fig. 3 around here

We examined performance on the recognition task by considering the distribution of choices as a function of whether the stimulus had been changing or not (see Figure 3). While there was a single correct response for the static stimuli, this was not the case for the changing stimuli since participants had been exposed to all intermediate images between the neutral and the emotional expression in that condition. In both cases however, we can compare the observed distribution to the flat distribution that would be produced by chance. For the static stimuli, the distribution of responses was different 
from chance $\left[\chi^{2}(4)=126.29 ; \mathrm{p}<0.0001\right]$. As expected, most participants $(52 \%)$ were able to correctly choose the image that they had actually been exposed to. For the changing stimuli, given that participants had actually been exposed for strictly equivalent durations to all morphs, one would expect to observe a flat distribution of choices if participants were simply guessing. However, this was not the case: The observed distribution differed both from chance $\left[\chi^{2}(4)=18.5 ; \mathrm{p}<0.001\right]$ and from the distribution obtained for static stimuli [paired-samples Wilcoxon test, $Z(35)=5.09 ; \mathrm{p}<0.0001$ ]. The mode for the static stimuli was $0-100 \%$ while the mode for the changing stimuli was 75 $25 \%$.

To determine whether subjects tended to choose one stimulus significantly more than another in both the static and changing conditions, we conducted Friedman ANOVAs. A first analysis was conducted on the static data. It revealed that choices were not equally distributed [Friedman test, $\left.\chi^{2}(\mathrm{n}=35, \mathrm{df}=4)=75.43 ; \mathrm{p}<0.00001\right]$. In addition, we compared the choices two by two with the mode $(0-100 \%)$ and found that the $0-100 \%$ stimulus was chosen significantly more often than the others (all ps<0.05). We also conducted a second Friedman ANOVA on the changing data [Friedman test, $\chi^{2}(\mathrm{n}=35$, $\mathrm{df}=4)=17.51 ; \mathrm{p}<0.001]$, again confirming that choices were not equally distributed. We also compared the choices two by two with the mode (75-25\%). This only revealed a significant difference with the $0-100 \%$ choice. Furthermore, we also compared the static and changing conditions for each choice. The 100-0\% morph was chosen significantly more often in the static than in the changing condition. For the $25-75 \%$ morph, there was a trend $(\mathrm{p}=0.068)$ for this morph to be chosen more often in the static than in the 
changing condition. All the other choices (i.e., 50-50\%, 75-25\%, and 100-0\%) were chosen significantly more often $(\mathrm{p}<0.05)$ in the changing than in the static condition.

To test for a possible effect of facial expression (emotional vs. neutral) on performance, we compared the choices for the neutral $(\mathrm{N})$ stimuli and emotional $(\mathrm{E})$ stimuli in the static condition [paired-sample Wilcoxon test, $Z(35)=2.09 ; p<0.03$ ]. This showed that observers tended to be more accurate when exposed to a static emotionally expressive face than when exposed to a neutral face. We also compared stimuli changing from Neutral $(\mathrm{N})$ to Emotional $(\mathrm{E})$ to stimuli changing from $\mathrm{E}$ to $\mathrm{N}$, but failed to find any significant difference [paired-sample Wilcoxon test, $Z(35)=0.04 ; p=0.96$ ].

Insert Fig. 4 around here

Finally, participants were significantly less confident in their choices for the changing than for the static stimuli [paired-sample Wilcoxon test, $\mathrm{Z}(35)=2.34$; $\mathrm{p}<$ 0.019] (see Figure 4).

\section{Discussion}

To assess what is represented when one is confronted with an undetected change, participants were exposed either to static or gradually changing pictures of faces for 12 seconds and asked to memorize them. Changes were applied to the emotional expression 
displayed by one of the actors, going from neutral to emotional for half of the stimuli and in the reverse direction for the other half. In a subsequent recognition test that followed each trial, participants were then shown 5 intermediate morphed pictures and were (1) asked to choose the picture they had just seen, and (2) asked to express their confidence in their decision.

A first result showed that quite a high proportion of subjects failed to detect any change (71.4\%). This is a rather high proportion considering (1) that observers had been instructed to focus on the faces of the actors and to memorize them; (2) that facial expressions are highly salient stimuli that are socially relevant in all cultures (Ekman \& Friesen, 1971).

Second, the recognition test showed that unaware participants' choices were different from chance, both for static and changing stimuli. Indeed, their responses were not equally distributed across the 5 intermediate pictures. This suggests that participants had stored information and had formed a representation of the stimuli even when these stimuli were changing. Moreover, the pattern of responses was different for the static and for the changing stimuli, as indicated by the fact that the modes of the distributions are different. This suggests that observers do not report the same representation when exposed to static vs. changing stimuli,. More precisely, the pattern of responses associated with changing stimuli (the mode for changing stimuli being on the " $75-25 \%$ " morph) suggests a recency rather than a primacy effect since subjects tended to remember what they had been exposed to most recently. However, they did not choose the final 
stimulus either. Two by two comparisons showed that in the case of static stimuli, the mode $(0-100 \%)$ was chosen significantly more often than the other alternatives, while for the changing condition, the mode $(75-25 \%)$ was only significantly different from the 0 $100 \%$ alternative. Nonetheless, our results clearly show that there is more of a recency than of a primacy effect in the changing condition, and that the distribution of responses is not flat.

A third result concerns the effects of emotional expression on participant's choices. In the static condition, participants were more accurate when exposed to a static emotionally expressive face than when exposed to a neutral face, while there was no difference in choices between stimuli that gradually changed from $\mathrm{N}$ to $\mathrm{E}$ and from $\mathrm{N}$ to $\mathrm{E}$ in the changing condition.

Finally, we observed a difference in the confidence judgments associated with decisions concerning static or changing stimuli. Participants expressed lower confidence in their recognition decisions after having been exposed to changing stimuli than to static stimuli. This suggests that while unaware that the stimuli were changing, participants were nevertheless somewhat aware that "something was off" with those stimuli. This indicates that they somehow knew that their representation was less accurate, but were not aware that this lack of accuracy was actually attributable to the variation of the stimuli themselves. Indeed, observers did not attribute the source of their uncertainty to the stimuli, but to their own experience of these stimuli. 
This study therefore suggests that an implicit update of visual memory seems to be at play when observers are exposed to a gradually changing stimulus. Our data also suggest that undetected changes in visual displays can influence both subsequent decisions as well as participant's confidence in these decisions.

However, it is important to note that an alternative account ${ }^{3}$ of the distribution observed in the changing condition is possible. First, observers might just be guessing more in the changing condition than in the static condition because the changing condition is inherently more difficult (since all alternatives had in fact been presented). This would, without further assumptions, produce a tendency for observers who guess to simply choose the average of the choices they are confronted with. Second, observers might just remember best what they have been exposed to last (the recency effect). These two mechanisms combined would then explain the skewed distribution of choices we observed based exclusively on a combination of guessing and recency. This hypothesis is further supported by the fact that observers are less confident in their response in the changing condition. By this account, thus, observers might not retain representations of the intermediate stages of the morph, but rather simply be more uncertain about what they have seen. Further research is clearly necessary to further explore this important issue. Nevertheless, regardless of which account is correct, our results indicate that observers unwittingly exposed to changing stimuli select what they think they have seen but (1) they do not choose at random and (2) their choices are made with lower confidence than in the static condition. It is striking that people's choices can be different

\footnotetext{
${ }^{3}$ We thank an anonymous reviewer for suggesting this alternative account.
} 
in the two conditions (static vs. changing) in spite of the fact that they all report identical subjective states with respect to change detection (i.e., all observers included in our analyses report not having perceived the change).

Finally, we note that our findings are in line with Hollingworth and Henderson (2004)'s threshold model for the conscious detection of change. According to these authors, the visual system is continuously actively acquiring information about the world through fixations and saccades of the eyes, but also thanks to head and body movements. In this context, the visual system's efficiency at detecting changes - surely one of its major functions - depends on its ability to filter out small internal errors. If our brain were to interpret any small discrepancy between two fixations as reflecting a change in the environment, there would be too many false alarms. For instance, if head and body movements were not perfectly compensated for during a blink, the visual system might attribute the resulting discrepancy to a change in the external world rather than to selfgenerated movement. This is in fact what happens when one presses on one's eyeball, so fooling the visual system into attributing the resulting changes to the external world rather than to the self-generated but highly unusual motion. A threshold system that requires that the difference between "views" reaches sufficient strength to cross a threshold allows the system to be resistant to such small internal errors. Gradual change stimuli fool the visual system into attributing subthreshold changes to internal error rather than to external events. This model thus suggests that the visual system actually detects small changes, but that such changes fail to be consciously represented as external changes and are hence dismissed. A threshold mechanism for change detection also 
explains why change blindness may occur despite accurate visual representations of the scene. Similar threshold accounts of change detection that might explain implicit change detection have already been suggested (Laloyaux et al., 2006) based on visual short term memory (VSTM) models that postulate noisy representations of the visual world (Baldassi \& Burr, 2000; Wilken \& Ma, 2004).

To conclude, our results suggest that people's representations of changing stimuli, as assessed through both a recognition task and through confidence judgments, is influenced by changing information in spite of the fact that people remained unaware that the stimuli had changed.

\section{Acknowledgments}

CL and CD are Research Fellows with the National Fund for Scientific Research (Belgium). AC is a research director with the same institution. This work was supported by an institutional grant from the Université Libre de Bruxelles and by Concerted Research Action 06/11-342 titled "Culturally modified organisms: What it means to be human in the age of culture", financed by the Ministère de la Communauté Française Direction Générale l'Enseignement non obligatoire et de la Recherche scientifique (Belgium). We thank the referees for their useful comments. 


\section{References}

Angelone, B. L., Levin, D. T., \& Simons, D. J. (2003). The relationship between change detection and recognition of centrally attended objects in motion pictures. Perception, 32(8), 947-962.

Auvray, M., \& O'Regan, J. K. (2003). L'influence des facteurs sémantiques sur la cécité au changement progressif dans les scènes visuelles. Année psychologique, 103(1), 9-32.

Baldassi, S., \& Burr, D. C. (2000). Feature-based integration of orientation signals in visual search. Vision Research, 40(10-12), 1293-1300.

Beck, M. R., \& Levin, D. T. (2003). The role of representational volatility in recognizing pre- and postchange objects. Perception and Psychophysics, 65(3), 458-468.

Becker, M. W., Pashler, H., \& Anstis, S. M. (2000). The role of iconic memory in change-detection tasks. Perception, 29(3), 273-286.

David, E., Laloyaux, C., Devue, C., \& Cleeremans, A. (in press). Change blindness to gradual changes in facial expressions. Psychologica Belgica.

Ekman, P., \& Friesen, W. V. (1971). Constants across cultures in the face and emotion. Journal of Personnality and Social Psychology, 17(2), 124-129.

Fernandez-Duque, D., Grossi, G., Thornton, I. M., \& Neville, H. J. (2003). Representation of change: Separate electrophysiological markers of attention, awareness, and implicit processing. Journal of Cognitive Neuroscience, 15(4), 491-507.

Fernandez-Duque, D., \& Thornton, I. M. (2000). Change detection without awareness: Do explicit reports underestimate the representation of change in the visual system? Visual Cognition, 7(1-3), 323-344.

Fernandez-Duque, D., \& Thornton, I. M. (2003). Explicit mechanisms do not account for implicit localization and identitication of change: An empirical reply to Mitroff et al. (2002). Journal of Experimental Psychology-Human Perception and Performance, 29(5), 846-858.

Hollingworth, A. (2003). Failures of retrieval and comparison constrain change detection in natural scenes. Journal of Experimental Psychology-Human Perception and Performance, 29(2), 388-403.

Hollingworth, A. (2004). Constructing visual representations of natural scenes: The roles of short- and long-term visual memory. Journal of Experimental PsychologyHuman Perception and Performance, 30(3), 519-537.

Hollingworth, A., \& Henderson, J. M. (2002). Accurate visual memory for previously attended objects in natural scenes. Journal of Experimental Psychology-Human Perception and Performance, 28(1), 113-136.

Hollingworth, A., \& Henderson, J. M. (2004). Sustained change blindness to incremental scene rotation: A dissociation between explicit change detection and visual memory. Perception \& Psychophysics, 66(5), 800-807.

Laloyaux, C., Destrebecqz, A., \& Cleeremans, A. (2006). Implicit change identification: a replication of Fernandez-Duque and Thornton (2003). Journal of Experimental Psychology-Human Perception and Performance, 32(6), 1366-1379. 
Landman, R., Spekreijse, H., \& Lamme, V. A. F. (2003). Large capacity storage of integrated objects before change blindness. Vision Research, 43(2), 149-164.

Levin, D. T., Momen, N., Drivdahl, S. B., \& Simons, D. J. (2000). Change blindness blindness: The metacognitive error of overestimating change-detection ability. Visual Cognition, 7(1-3), 397-412.

Levin, D. T., \& Simons, D. J. (1997). Failure to detect changes to attended objects in motion pictures. Psychonomic Bulletin \& Review, 4(4), 501-506.

Mitroff, S. R., Simons, D. J., \& Franconeri, S. L. (2002). The siren song of implicit change detection. Journal of Experimental Psychology-Human Perception and Performance, 28(4), 798-815.

Mitroff, S. R., Simons, D. J., \& Levin, D. T. (2004). Nothing compares 2 views: change blindness can occur despite preserved access to the changed information. Perception and Psychophysics, 66(8), 1268-1281.

O'Regan, J. K., \& Noe, A. (2001). A sensorimotor account of vision and visual consciousness. Behavioral and Brain Sciences, 24(5), 939-+.

Pashler, H. (1988). Familiarity and Visual Change Detection. Perception \& Psychophysics, 44(4), 369-378.

Phillips, W. A. (1974). On the distinction between sensory storage and short-term visual memory. Perception and Psychophysics, 16, 283-290.

Rensink, R. A. (2002). Change detection. Annual Review of Psychology, 53, 245-277.

Rensink, R. A., O'Regan, J. K., \& Clark, J. J. (2000). On the failure to detect changes in scenes across brief interruptions. Visual Cognition, 7(1-3), 127-145.

Rensink, R. A., ORegan, J. K., \& Clark, J. J. (1997). To see or not to see: The need for attention to perceive changes in scenes. Psychological Science, 8(5), 368-373.

Scott-Brown, K. C., Baker, M. R., \& Orbach, H. S. (2000). Comparison blindness. Visual Cognition, 7(1-3), 253-267.

Silverman, M. E., \& Mack, A. (2006). Change blindness and priming: When it does and does not occur. Consciousness and Cognition, 15(2), 409-422.

Simons, D. J. (2000). Current approaches to change blindness. Visual Cognition, 7(1-3), $1-15$.

Simons, D. J., Chabris, C. F., Schnur, T., \& Levin, D. T. (2002). Evidence for preserved representations in change blindness. Consciousness and Cognition, 11(1), 78-97.

Simons, D. J., Franconeri, S. L., \& Reimer, R. L. (2000). Change blindness in the absence of a visual disruption. Perception, 29(10), 1143-1154.

Simons, D. J., \& Levin, D. T. (1998). Failure to detect changes to people during a realworld interaction. Psychonomic Bulletin \& Review, 5(4), 644-649.

Simons, D. J., \& Levin, D. T. (2003). What makes change blindness interesting? Psychology of Learning and Motivation: Advances in Research and Theory: Cognitve Vision, Vol 42, 42, 295-322.

Tatler, B. W. (2001). Characterising the visual buffer: real-world evidence for overwriting early in each fixation. Perception, 30(8), 993-1006.

Thornton, I. M., \& Fernandez-Duque, D. (2000). An implicit measure of undetected change. Spatial Vision, 14(1), 21-44.

Thornton, I. M., \& Fernandez-Duque, D. (2002). Change blindness and transsaccadic integration. In J. hyönä, D. P. Munoz, W. Heide \& R. Radach (Eds.), Progress in Brain Research (Vol. 140). Amsterdam: Elsevier science. 
Velichkovsky, B. M., Dornhoefer, S. M., Kopf, M., Helmert, J., \& Joos, M. (2002). Change detection and occlusion modes in road-traffic scenarios. Transportation Research Part F, 5, 99-109.

Wilken, P., \& Ma, W. J. (2004). A detection theory account of change detection. Journal of Vision, 4(12), 1120-1135. 


\section{Figure captions}

Figure 1. Example of a scene used in this study in which all the pixels that change from Neutral to Emotional stimulus are highlighted. On average, the changing pixels represented $6.4 \%$ of the total surface of the image for expression changes.

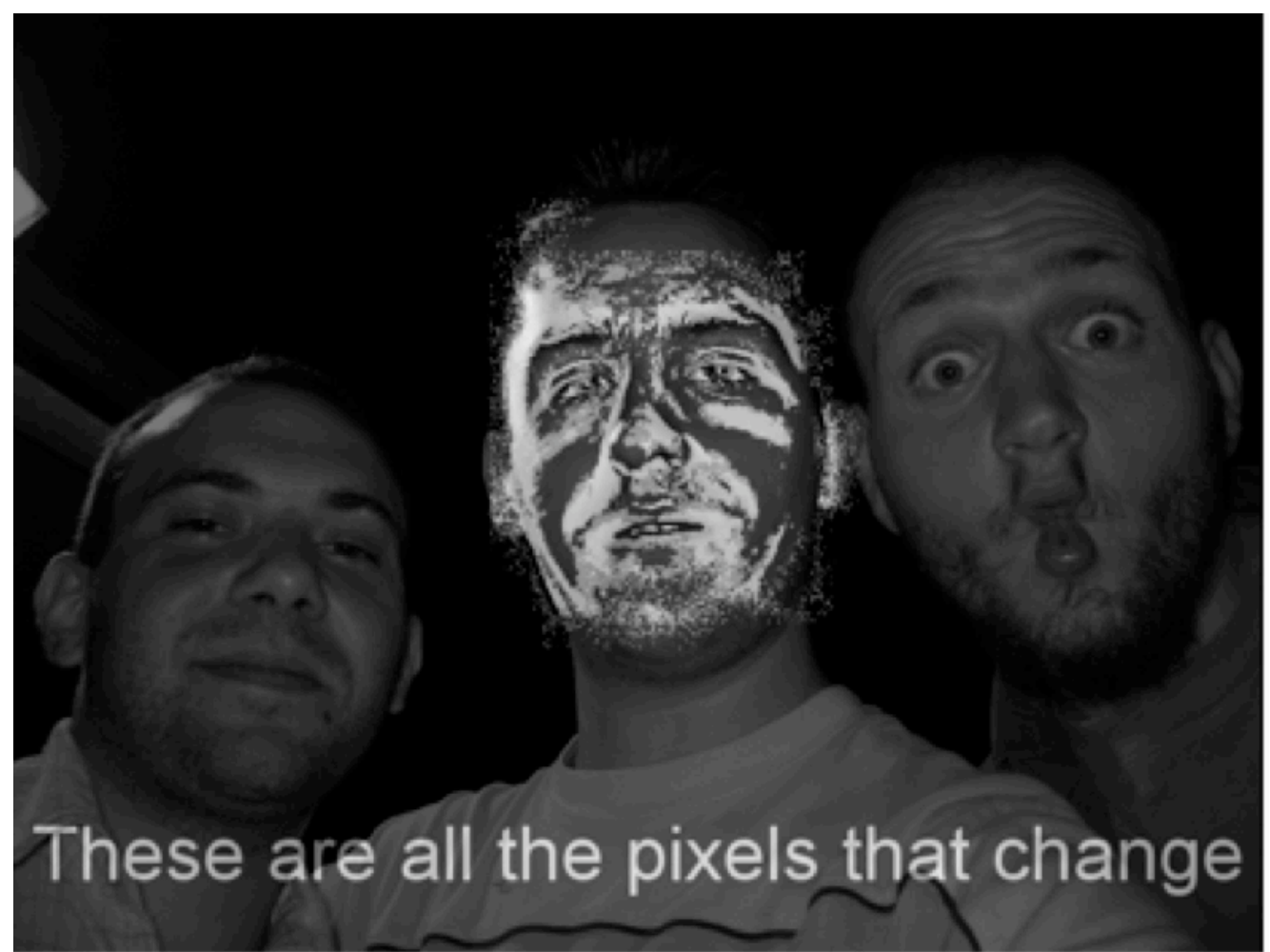


Figure 2. Time course of a single trial of Experiment 2. Observers were first exposed to either a static or to a changing scene for 12 seconds. They were then exposed to a forcedchoice recognition task. Finally, they were asked to rate their confidence in their recognition decisions.

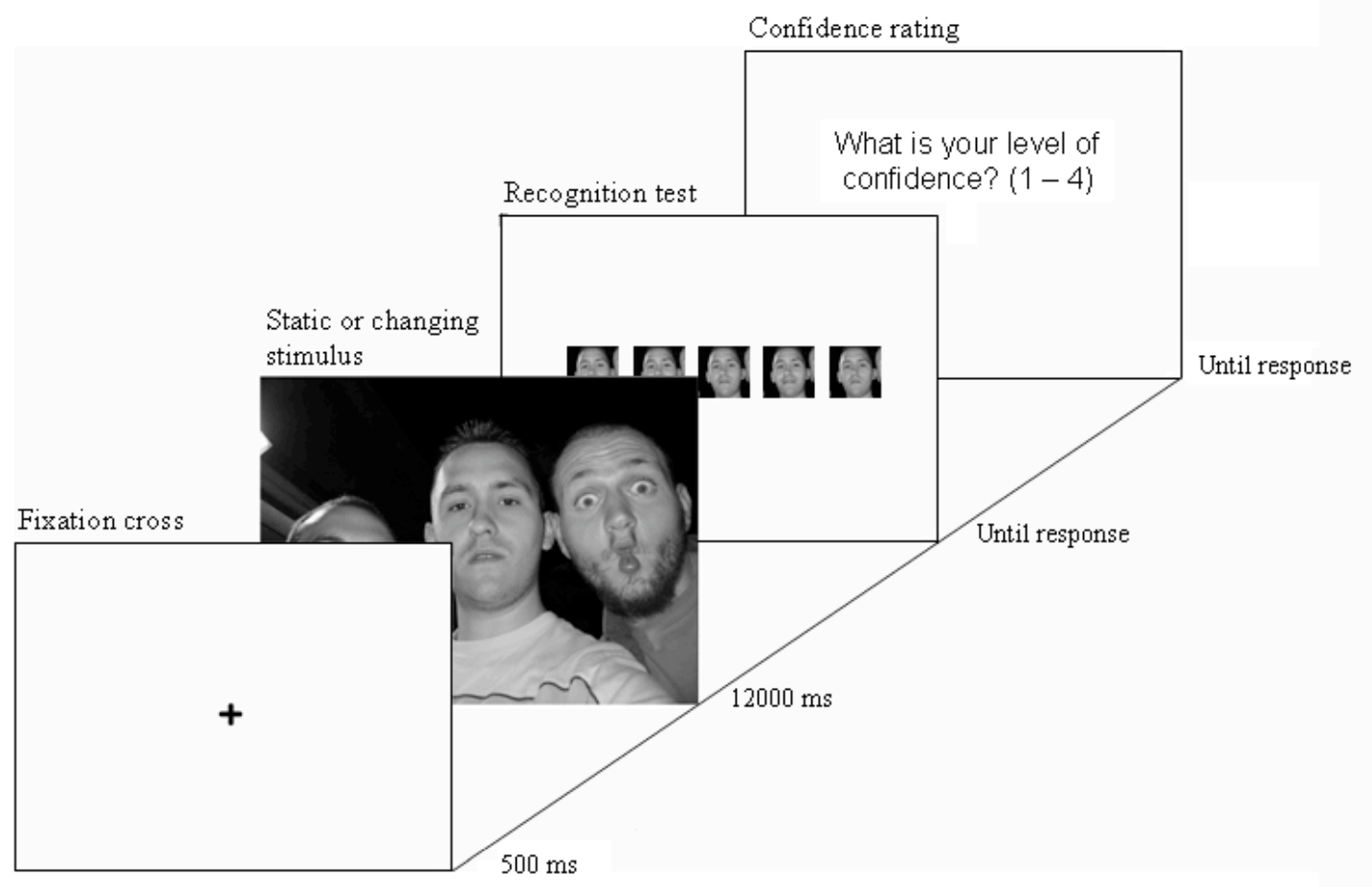


Figure 3. Sample stimuli and results of Experiment 2: Choice proportion for each morph presented in the forced choice recognition task for static and changing stimuli. The correct answer for static stimuli corresponded to the " $0-100 \%$ " image. In half of the cases, an emotional picture had been presented, in the other half of the cases, a neutral picture had been presented. For the changing stimuli, the beginning of the movie is represented by " $0-100 \%$ " and the end by " $100-0 \%$ " on the $\mathrm{X}$ axis. There is thus no correct answer in the changing condition. In half of the cases, the facial expression of the target changed from emotional to neutral while in the remaining half, the facial expression changed from neutral to emotional.

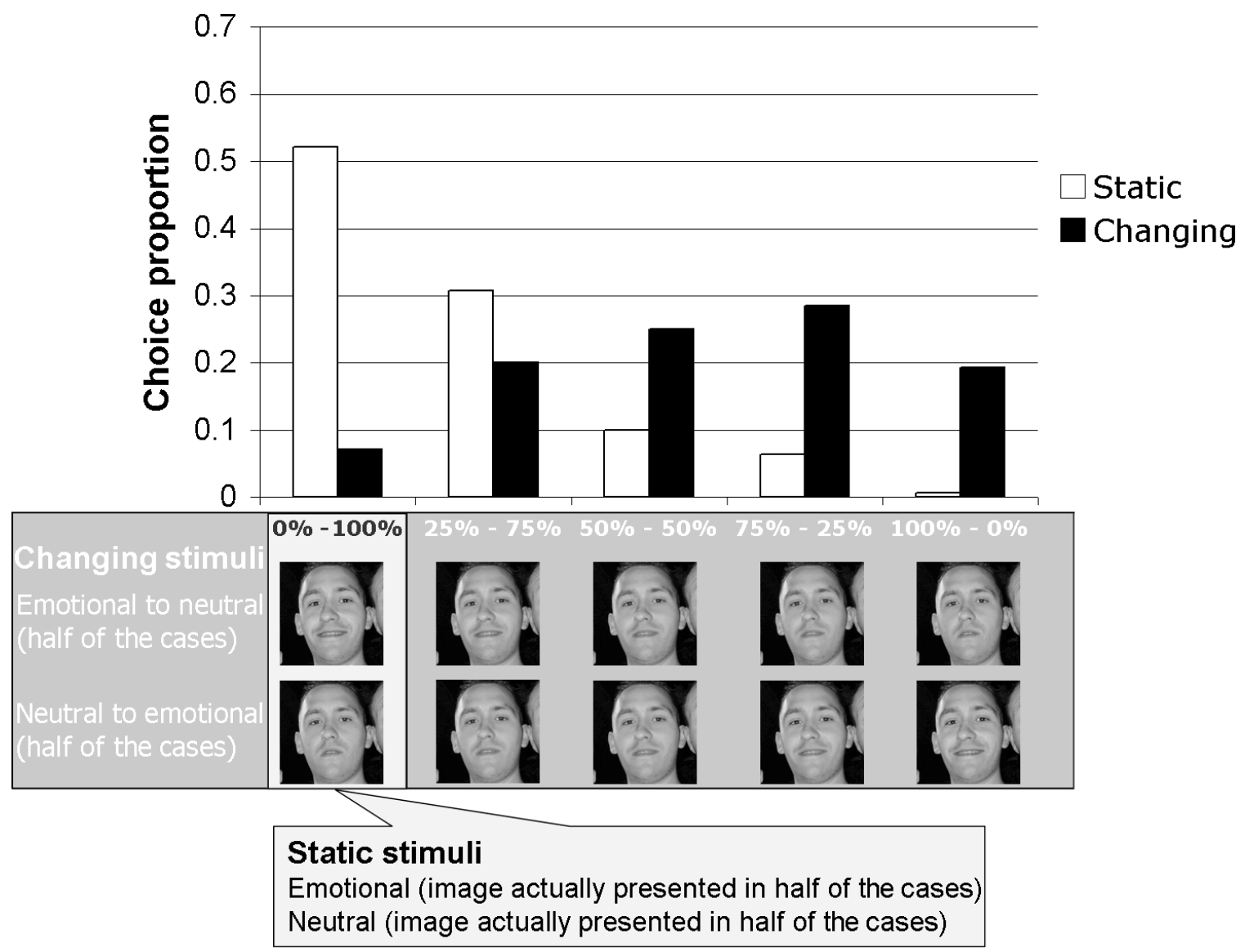


Figure 4. Results of Experiment 2: Confidence ratings for the static and changing stimuli. Observers expressed their confidence on a scale from 1 to 4 (see main text).

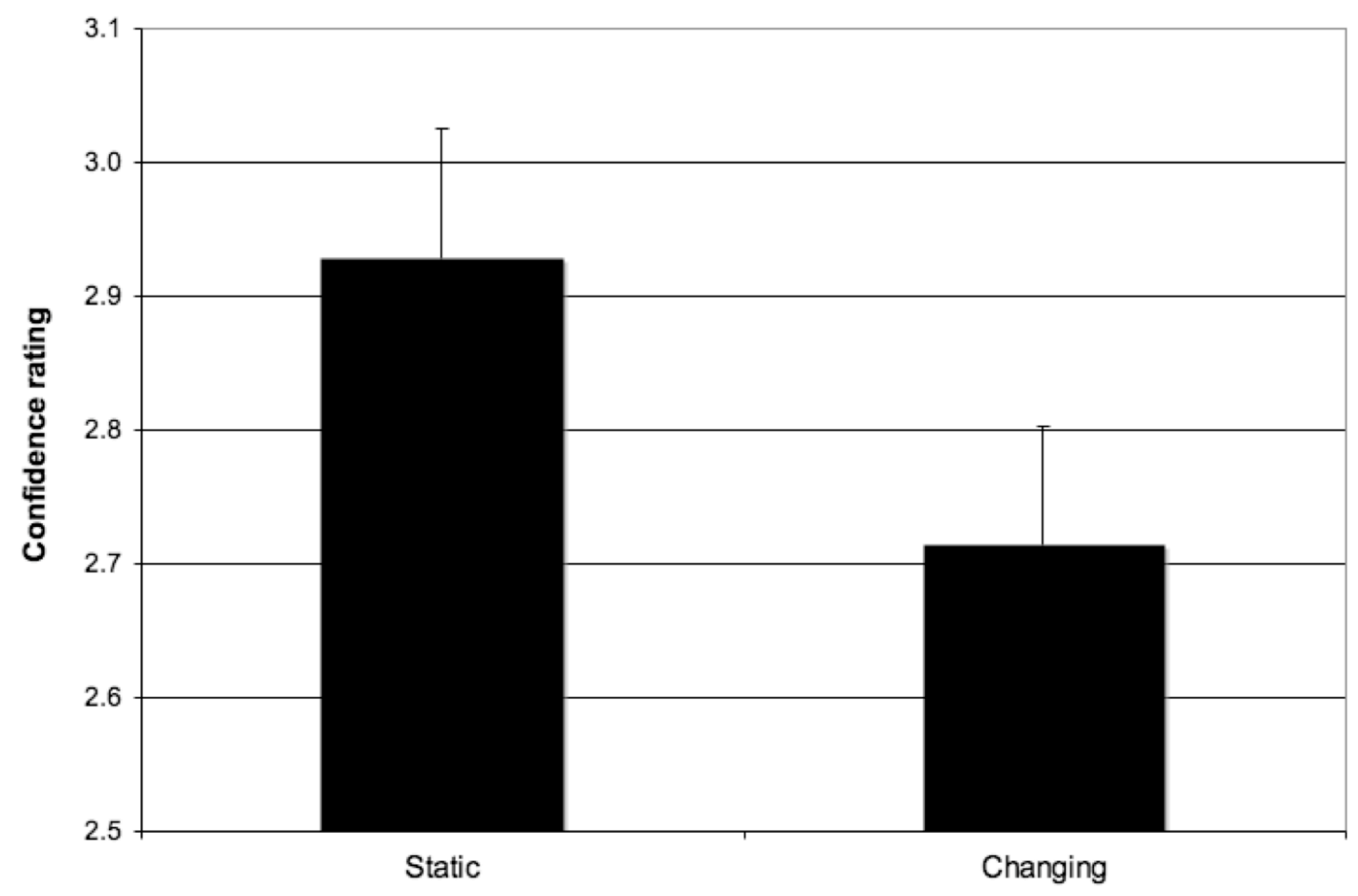

